\title{
Quantum Search on Graphene Lattices
}

\author{
Iain Foulger, Sven Gnutzmann, and Gregor Tanner \\ School of Mathematical Sciences, University of Nottingham, University Park, Nottingham NG7 2RD, United Kingdom \\ (Received 18 April 2013; revised manuscript received 10 July 2013; published 21 February 2014)
}

\begin{abstract}
We present a continuous-time quantum search algorithm on a graphene lattice. This provides the soughtafter implementation of an efficient continuous-time quantum search on a two-dimensional lattice. The search uses the linearity of the dispersion relation near the Dirac point and can find a marked site on a graphene lattice faster than the corresponding classical search. The algorithm can also be used for state transfer and communication.
\end{abstract}

Introduction.-Quantum walks [1,2] can provide polynomial and even exponential speed-up compared to classical random walks [3-6] and may serve as a universal computational primitive for quantum computation [7]. This has led to substantial interest in the theoretical aspects of this phenomenon, as well as in finding experimental implementations [8-13]. One of the most fascinating applications of quantum walks is their use in spatial quantum search algorithms first published for the search on the hypercube in Ref. [14]. Like Grover's search algorithm $[15,16]$ for searching an unstructured database, quantum walk search algorithms can achieve up to quadratic speed-up compared to the corresponding classical search. For quantum searches on $d$-dimensional square lattices, certain restrictions have been observed, however, depending on whether the underlying quantum walk is discrete [3] or continuous [17]. While effective search algorithms for discrete walks have been reported for $d \geq 2$ $[18,19]$, continuous-time quantum search algorithms on square lattices show speed-up compared to the classical search only for $d \geq 4$ [20]. This problem has been circumvented in Ref. [21], however, at the conceptual cost of adding internal degrees of freedom (spin) and a discrete Dirac equation.

Experimental implementations of discrete quantum walks need time-stepping mechanisms such as laser pulses $[8-11,13]$. Thus, in general, it is simpler to consider experimental realizations with continuous-time evolution. However, in the absence of internal degrees of freedom, no known search algorithm on lattices exists up to now in the physically relevant regime $d=2$ or 3 . Finding such an algorithm is highly topical due to applications in secure state transfer and communication across regular lattices, as demonstrated in Ref. [22].

Published by the American Physical Society under the terms of the Creative Commons Attribution 3.0 License. Further distribution of this work must maintain attribution to the author(s) and the published article's title, journal citation, and DOI.
We will show in the following that continuous-time quantum search in $2 \mathrm{D}$ is, indeed, possible. We will demonstrate that such a quantum search can be performed at the Dirac point in graphene. This is potentially of great interest, as graphene is now becoming available cheaply and can be fabricated routinely $[23,24]$. Performing quantum search and quantum state transfer on graphene provides a new way of channeling energy and information across lattices and between distinct sites. Graphene sheets have been identified as a potential single-molecule sensor $[25,26]$ being very sensitive to a change of the density of states near the Dirac point. This property is closely related to the quantum search effect described in this Letter.

Continuous-time quantum search algorithms take place on a lattice with a set of $N$ sites interacting via hopping potentials (usually between nearest neighbors only). Standard searches work at the ground-state energy which, due to the periodicity of the lattice, is related to quasimomentum $k=0$. After introducing a perturbation at one of the lattice sites, the parameters are adjusted such that an avoided crossing between the localized "defect" state and the ground state is formed. The search is now performed in this two-level subsystem [27]. Criticality with respect to the dimension is reached when the gap at the avoided crossing and the eigenenergy spacing near the crossing scale in the same way with $N$.

Continuous-time quantum walks [17] operate in the position (site) space. If the states $|j\rangle$ represent the sites of the lattice, the Schrödinger equation governing the probability amplitudes $\alpha_{j}(t)=\langle j \mid \psi(t)\rangle$ is given by

$$
\frac{d}{d t} \alpha_{j}(t)=-i \sum_{l=1}^{N} H_{j l} \alpha_{l}(t),
$$

where the Hamiltonian $\mathbf{H}=\varepsilon_{D} \mathbf{I}+v \mathbf{A}$ is of tight-binding type where $\mathbf{A}$ is the adjacency matrix of the lattice and $\mathbf{I}$ is the identity matrix, $\epsilon_{D}$ is the on-site energy, and $v$ is the strength of the hopping potential. In Ref. [20], the walk Hamiltonian was set to be the discrete Laplacian where $v=-1$ and $\epsilon_{D}$ is the coordination number of the lattice. 
A marked site is then introduced by altering the on-site energy of that site. The system is initialized at $t=0$ in the ground state of the unperturbed lattice leading to an effective search for $d \geq 4$. For the search based on the discretized Dirac operator [21], an additional spin degree of freedom is introduced. This gives optimum search times for lattices with dimension $d \geq 3$ and a search time of $O(\sqrt{N} \ln N)$ for $d=2$ recovering the results for discrete time walks [18]. We note that $d=2$ is the critical dimension in the discrete case independent of the lattice structure; thus, one finds an $O(\sqrt{N} \ln N)$ also for discrete time walks on graphene [19].

The lack of speed-up for continuous search algorithms in two dimensions can be overcome by making two adjustments: (i) the avoided crossing on which the search operates is moved to a part of the spectrum with a linear dispersion relation; (ii) the local perturbation is altered in order to couple a localized perturber state and the lattice state in the linear regime. The first point is addressed by considering graphene lattices with the well-known linear dispersion curves near the Dirac point. The perturbation at the marked site is achieved by locally changing the hopping potential (instead of changing the on-site energy as in Refs. [20,21]). We start by giving an introductory account of basic properties of the graphene lattice and its band structure [23,24].

Review of graphene.-The graphene (or honeycomb) lattice is bipartite with two triangular sublattices labeled $A$ and $B$. The position of a cell in the lattice is denoted by $\underline{R}=\alpha \underline{a}_{1}+\beta \underline{a}_{2}$ where $\alpha$ and $\beta$ are integers and $\underline{a}_{1(2)}$ are basis vectors of the lattice (see Fig. 1). States on the two sites within one cell will be denoted by $|R\rangle^{A(B)} \equiv|\alpha, \beta\rangle^{A(B)}$. The corners of the Brillouin zone (see Fig. 1) are denoted $\underline{K}$ and $\underline{K}^{\prime}$ and the primitive cell contains two of these points.

The solution for the tight-binding Hamiltonian on graphene as described above is well known [23,24] and leads to the dispersion relation

$$
\begin{aligned}
\epsilon(\underline{k})= & \epsilon_{D} \\
& \pm v \sqrt{1+4 \cos ^{2}\left(\frac{k_{x} a}{2}\right)+4 \cos \left(\frac{k_{x} a}{2}\right) \cos \left(\frac{\sqrt{3} k_{y} a}{2}\right)}
\end{aligned}
$$
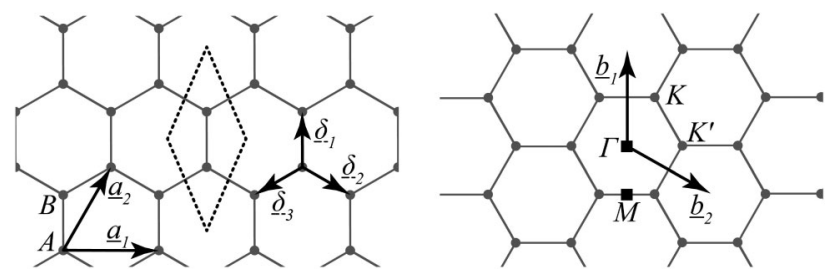

FIG. 1. Left: Graphene with lattice vectors $\underline{a}_{1 / 2}$, translation vectors $\underline{\delta}_{i}$, and unit cell (dashed lines). Right: Reciprocal lattice with basis vectors $\underline{b}_{1 / 2}$, symmetry points $\underline{\Gamma}, \underline{K}, \underline{K^{\prime}}, \underline{M}$, and first Brillouin zone (hexagon). depicted in Fig. 2 for an infinite graphene lattice. It is, indeed, linear near the Dirac points $\underline{K}$ and $\underline{K}^{\prime}$ at the energy $\epsilon_{D}$ where the conduction and valence bands meet. Around the Dirac points, the dispersion relation $\epsilon(\underline{k})$ can be approximated by

$$
\epsilon(\underline{k}) \approx \epsilon_{D} \pm v a \frac{\sqrt{3}}{2} \sqrt{\delta k_{x}^{2}+\delta k_{y}^{2}}=\epsilon_{D} \pm v a \frac{\sqrt{3}}{2}|\delta k| .
$$

In the following, we will consider finite graphene lattices with periodic boundary conditions, i.e., $|\Psi\rangle=$ $\sum_{\alpha=1}^{m} \sum_{\beta=1}^{n}\left(\psi_{\alpha, \beta}^{A}|\alpha, \beta\rangle^{A}+\psi_{\alpha, \beta}^{B}|\alpha, \beta\rangle^{B}\right) \quad$ with $\quad \psi_{\alpha, \beta}^{A(B)}=$ $\psi_{\alpha+m, \beta}^{A(B)}=\psi_{\alpha, \beta+n}^{A(B)}$. This simplifies the analysis allowing us to focus on the relevant features of the search by avoiding boundary effects. The general description does not change for other boundary conditions; the localization amplitude on the marked site, however, becomes site dependent in a nontrivial way. Understanding this dependency is not essential in the context of this Letter.

We denote $\underline{S}=m \underline{a}_{1}+n \underline{a}_{2}$ as the vector describing the spatial dimensions of the lattice. Using Bloch's theorem [28], the momentum is quantized as

$$
k_{x}=\frac{2 \pi p}{m a} \quad k_{y}=\frac{1}{\sqrt{3}}\left(\frac{4 \pi q}{n a}-k_{x}\right)
$$

where $p \in\{0,1, \ldots, m-1\}, q \in\{0,1, \ldots, n-1\}$, and the spectrum (2) becomes discrete. In what follows, for simplicity, we have assumed that our lattice is square in the number of cells, that is, that $m=n=\sqrt{\frac{N}{2}}$. Fourfold degenerate states with energy $\epsilon_{D}$ and wave numbers exactly on the Dirac points $\underline{K}$ and $\underline{K}^{\prime}$ exist if $m$ and $n$ are some multiples of 3 . We assume this in the following for simplicity. In the general case, one needs to consider the states closest to the Dirac energy which gives a more

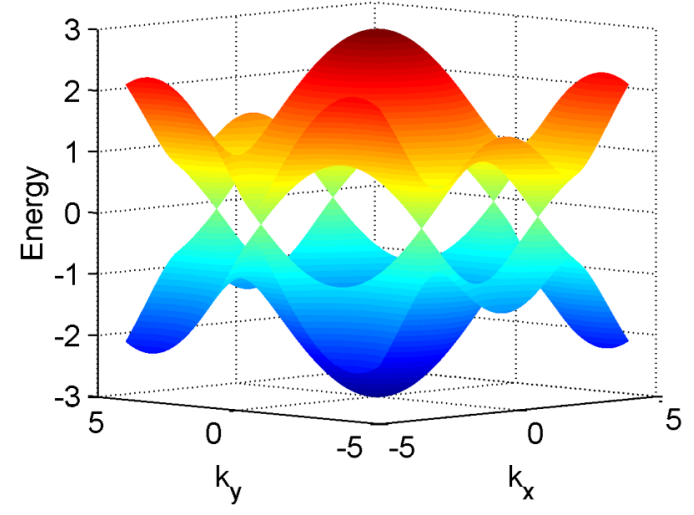

FIG. 2 (color online). Dispersion relation for infinite graphene sheet $\left(\epsilon_{D}=0\right)$. 
complex theoretical analysis while essential signatures do not change.

Quantum search.-Setting up a continuous-time search by changing the on-site energy of the marked site as done in Ref. [20] does not work for graphene. Using the ground state as the starting state fails for the same reason as it fails for rectangular lattices in $d=2$ or 3 as the dispersion relation is quadratic near the ground state, see Fig. 2. Alternatively, moving the search to the Dirac point implies constructing an avoided crossing between a localized perturber state and a Dirac state. As the Dirac energy coincides with the on-site energy $\epsilon_{D}$, this leads to the condition that the on-site energy perturbation must vanish at the crossing, which brings us back to the unperturbed lattice.

We, therefore, mark a given site by changing the hopping potentials between the site and its nearest neighbors. Focusing on a symmetric choice of the perturbation and setting $\epsilon_{D}=0$ for convenience, we obtain the (search) Hamiltonian

$$
\mathbf{H}_{\gamma}=-\gamma \mathbf{A}+\mathbf{W} .
$$

Here, $\mathbf{W}$ denotes the perturbation changing the hopping potential to and from the marked site $\left(\alpha_{0}, \beta_{0}\right)^{A}$, which has been chosen to be on the $A$ lattice, that is,

$$
\mathbf{W}=\sqrt{3}\left|\alpha_{0}, \beta_{0}\right\rangle^{A}\langle\ell|+\sqrt{3}| \ell\rangle\left\langle\alpha_{0},\left.\beta_{0}\right|^{A} .\right.
$$

The state $|\ell\rangle$ denotes the symmetric superposition of the three neighbors of the marked site, that is

$$
|\ell\rangle=\frac{1}{\sqrt{3}}\left(\left|\alpha_{0}, \beta_{0}\right\rangle^{B}+\left|\alpha_{0}, \beta_{0}-1\right\rangle^{B}+\left|\alpha_{0}+1, \beta_{0}-1\right\rangle^{B}\right) .
$$

At $\gamma=1$, the perturbation corresponds to a hopping potential $v=0$ between the site $\left(\alpha_{0}, \beta_{0}\right)^{A}$ and its neighbors, effectively removing the site from the lattice. It is this perturbation strength which is important in the following. Experimentally, such a perturbation is similar to graphene lattices with atomic vacancies as they occur naturally in the production process [29]; in microwave analogs of graphene, as discussed in Ref. [30], this can be realized by removing single sites from the lattice.

The effect of marking (or perturbing) the graphene Hamiltonian can be seen numerically in the parametric behavior of the spectrum of $\mathbf{H}_{\gamma}$ as a function of $\gamma$, see Fig. 3 for the case $n=m=12$. Note that $\mathbf{W}$ is a rank-two perturbation which creates two perturber states. These states start to interact with the spectrum of the unperturbed graphene lattice from $\gamma \approx 0.5$ onwards working their way through to a central avoided crossing at $\gamma=1, E=0$. Below we will show how the avoided crossing can be used for searching; note that the parameter dependence of the

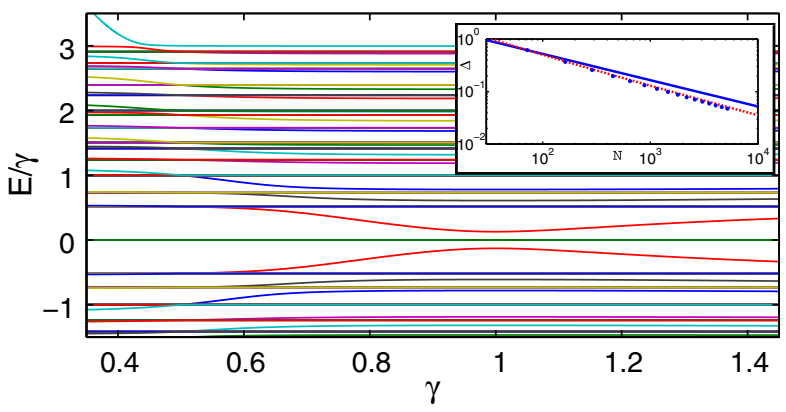

FIG. 3 (color online). Spectrum $\mathbf{H}_{\gamma}$ in Eq. (5) as a function of $\gamma$ for a $12 \times 12$ cell torus $(N=288)$. The spectrum is symmetric around $\epsilon_{D}=0$. Inset: Scaling of the gap $\Delta=\tilde{E}_{+}-\tilde{E}_{-}$(dots) and curves $c_{1} / \sqrt{N}$ (solid blue), $c_{2} / \sqrt{N \log N}$ (dashed red) for comparison.

avoided crossing $(\gamma=1)$ is evident from the tight-binding Hamiltonian $H$ in Eq. (5). In a realistic setup, the perturbation needs to be fine-tuned in general to be in resonance with an eigenstate of the (unperturbed) system near the Dirac point.

At the avoided crossing, there are altogether six states close to the Dirac energy: the two perturber states and the four degenerate Dirac states,

$$
\begin{aligned}
|K\rangle^{A(B)} & =\sqrt{\frac{2}{N}} \sum_{\alpha, \beta} e^{i \frac{2 \pi}{3}(\alpha+2 \beta+2 \sigma)}|\alpha, \beta\rangle^{A(B)}, \\
\left|K^{\prime}\right\rangle^{A(B)} & =\sqrt{\frac{2}{N}} \sum_{\alpha, \beta} e^{i \frac{2 \pi}{3}(2 \alpha+\beta)}|\alpha, \beta\rangle^{A(B)},
\end{aligned}
$$

where $\sigma=1(\sigma=0)$ for states on the $B(A)$ lattice and $N=2 \mathrm{~nm}$ is the number of sites in the lattice. One finds directly $\mathbf{W}|K\rangle^{B}=\mathbf{W}\left|K^{\prime}\right\rangle^{B}=0$; that is, Dirac states on the $B$ lattice do not interact with an $A$-type perturbation for all $\gamma$. Furthermore, at $\gamma=1$, the marked site is disconnected from the lattice; the marked state $\left|\alpha_{0}, \beta_{0}\right\rangle$ is therefore an eigenvector of $\mathbf{H}_{\gamma=1}$ with eigenvalue $E=0$.

Thus, the avoided crossing involves only the two Dirac states $|K\rangle^{A},\left|K^{\prime}\right\rangle^{A}$ and one perturber state $|\tilde{\ell}\rangle$. Neglecting the interaction of the perturbation with the rest of the spectrum at the avoided crossing, we set $|\tilde{\ell}\rangle \approx|\ell\rangle$ [see Eq. (7)] and use this to reduce the full Hamiltonian locally in terms of the three-dimensional basis $\left\{|K\rangle^{A},\left|K^{\prime}\right\rangle^{A},|\ell\rangle\right\}$. The reduced Hamiltonian takes the form

$\tilde{\mathbf{H}}=\sqrt{\frac{6}{N}}\left[\begin{array}{ccc}0 & 0 & e^{-i(2 \pi / 3)\left(\alpha_{o}+2 \beta_{o}\right)} \\ 0 & 0 & e^{-i(2 \pi / 3)\left(2 \alpha_{o}+\beta_{o}\right)} \\ e^{i(2 \pi / 3)\left(\alpha_{o}+2 \beta_{o}\right)} & e^{i(2 \pi / 3)\left(2 \alpha_{o}+\beta_{o}\right)} & 0\end{array}\right]$,

with eigenvalues $\tilde{E}_{ \pm}= \pm 2 \sqrt{\frac{3}{N}}, \tilde{E}_{0}=0$, and eigenvectors 


$$
\begin{gathered}
\left|\tilde{\psi}_{ \pm}\right\rangle=\frac{1}{2}\left(e^{-i(2 \pi / 3)\left(\alpha_{o}+2 \beta_{o}\right)}|K\rangle^{A}\right. \\
\left.+e^{-i(2 \pi / 3)\left(2 \alpha_{o}+\beta_{o}\right)}\left|K^{\prime}\right\rangle^{A} \pm \sqrt{2}|\ell\rangle\right) \\
\left|\tilde{\psi}_{0}\right\rangle=\frac{1}{\sqrt{2}}\left(e^{-i(2 \pi / 3)\left(\alpha_{o}+2 \beta_{o}\right)}|K\rangle^{A}-e^{-i(2 \pi / 3)\left(2 \alpha_{o}+\beta_{o}\right)}\left|K^{\prime}\right\rangle^{A}\right) .
\end{gathered}
$$

For searching the marked site $\left(\alpha_{0}, \beta_{0}\right)^{A}$, the system is initialized in a delocalized starting state involving a superposition of Dirac states. This state will then rotate into a state localized on the neighbors of the marked site. The search is initialized in the optimal starting state

$$
\begin{aligned}
|s\rangle & =\frac{1}{\sqrt{2}}\left(\left|\tilde{\psi}_{+}\right\rangle+\left|\tilde{\psi}_{-}\right\rangle\right) \\
& =\frac{e^{-i(2 \pi / 3)\left(\alpha_{0}+2 \beta_{0}\right)}}{\sqrt{2}}\left(|K\rangle^{A}+e^{-i(2 \pi / 3)\left(\alpha_{0}-\beta_{0}\right)}\left|K^{\prime}\right\rangle^{A}\right),
\end{aligned}
$$

which still depends on the perturbed site. A lack of knowledge of $\left(\alpha_{0}, \beta_{0}\right)$ leads, however, only to an $N$-independent overhead, see the discussion below. Letting $|s\rangle$ evolve in time with the reduced Hamiltonian (9), we obtain

$$
\begin{aligned}
|\psi(t)\rangle & =e^{-i \tilde{\mathbf{H}} t}|s\rangle=\frac{1}{\sqrt{2}}\left(e^{-i \tilde{E}_{+} t}\left|\tilde{\psi}_{+}\right\rangle+e^{-i \tilde{E}_{-} t}\left|\tilde{\psi}_{-}\right\rangle\right) \\
& =\cos \left(\tilde{E}_{+} t\right)|s\rangle-i \sin \left(\tilde{E}_{+} t\right)|\ell\rangle,
\end{aligned}
$$

that is, the system rotates from $|s\rangle$ to $|\ell\rangle$ in time $t=(\pi / 4) \sqrt{\frac{N}{3}}$. We find a $\sqrt{N}$ speed-up for the search on graphene. This, together with the linear dispersion relation near the Dirac point, where the spacing between successive eigenenergies scales like $O(1 / \sqrt{N})$, makes the search on this 2D lattice possible. In contrast to the algorithms described in Refs. [20,21], the system localizes here on the neighbors of the marked site; the marked site can be found by three additional direct queries. Furthermore, the initial starting state is not the uniform state here but the state $|s\rangle$ in Eq. (12). To construct this initial state uniquely requires some information about the site that is being searched for. Without this knowledge, one has three possible optimal initial states for an $A$-type perturbation, as can be seen from Eq. (12). The same applies for marking a $B$-type site, so in total there are six possible optimal starting states. As these states are not orthogonal, this increases the number of runs for a successful search by a factor of 4. The additional overhead is independent of $N$, and, thus, does not alter the scaling with system size. In an experiment, one may have little control about how the system is excited at the Dirac energy, so the initial state will be in a more or less arbitrary superposition of all four Dirac states. The search is then not optimal but runs with a

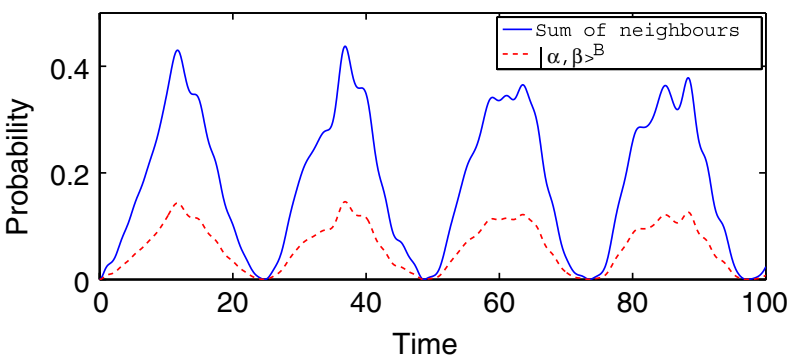

FIG. 4 (color online). Search on $12 \times 12$ cell graphene lattice with starting state $|s\rangle$. For tori with $m=n$, the dynamics at each neighboring site is the same, so only one is shown.

success probability that is, on average, again reduced by a factor of $1 / 4$.

Figure 4 shows a numerically obtained quantum search initialized in $|s\rangle$ and evolving under the full search Hamiltonian. As expected from the analysis on the reduced Hamiltonian, the state localizes on the three neighboring sites with a probability of about $45 \%$, which is 2 orders of magnitude larger than the average probability $100 / N \%$, here roughly $0.5 \%$. The search does not reach $100 \%$ due to the fact that the actual localized state $|\tilde{\ell}\rangle$ extends beyond the nearest neighbors of the marked site, so $\langle\tilde{\ell} \mid \ell\rangle=O(1)<1$.

Our reduced model neglects contributions from the rest of the spectrum; like for other discrete and continuous-time walks at the critical dimension [17-21], these contributions give $\ln N$ corrections [such as the $O(1 / \sqrt{N \ln (N)})$ scaling of the gap at the avoided crossing shown in the inset of Fig. 3]. These logarithmic corrections have been derived in Ref. [31] by going beyond the reduced three-state model, see also Ref. [20]. The relevant exact eigenenergies $E_{+}=$ $-E_{-}$at the avoided crossing satisfy the resolvent condition

$$
F\left(E_{ \pm}\right)=\frac{\sqrt{3}}{N} \sum_{\underline{k}}\left[\frac{1}{E_{ \pm}-\epsilon(\underline{k})}+\frac{1}{E_{ \pm}+\epsilon(\underline{k})}\right]=0,
$$

with $\epsilon(k)>0$ the eigenenergies of the unperturbed system at quasimomenta $k$ given in Eq. (4). Expanding $F\left(E_{+}\right)=4 \sqrt{3} /\left(N E_{+}\right)-\sum_{n=1}^{\infty} I_{2 n} E_{+}^{2 n-1}$, one finds $I_{2}=$ $O(\ln N)$ and $I_{2 k}=O\left(N^{k-1}\right)$ for $k \geq 2$, see Ref. [31] for details. The scaling of the gap follows then directly. The localization time scales inversely proportional to the gap, that is, $T=O(\sqrt{N \ln (N)})$; one also obtains that the return amplitude drops like $O(1 / \sqrt{\ln N})$.

We note in passing that our search algorithm can-like all quantum searches-be used for quantum communication and state transfer. Following Ref. [22], our continuoustime search can be used to send signals between different sites by adding an additional perturbation to the lattice. The quantum system is then initialized in a state localized on one of the perturbed sites and the system oscillates between 
states localized on the perturbations. We find that the mechanism works best when both perturbations are on the same sublattice. Because of the nature of the coupling between the $A$ and $B$ sublattices and the fact that the localized perturber states live (mostly) on one sublattice, signal propagation between perturbations on different sublattices takes place over a much longer time scale.

Discussion.-Continuous-time quantum search can be performed effectively on a 2D lattice without internal degrees of freedom by running the search at the Dirac point in graphene. We find that our search succeeds in time $T=O(\sqrt{N \ln (N)})$ with probability $O(1 / \ln N)$ [31]. This is the same time complexity found in Refs. $[18,19]$ for discrete-time searches and in Ref. [21] for continuoustime searches. To boost the probability to $O(1), O(\ln N)$ repetitions are required giving a total time $T=O\left(\sqrt{N} \ln { }^{(3 / 2)} N\right)$. Amplification methods [32-34] may be used to reduce the total search time further.

For simplicity of the analysis, we have focused here on perturbations which alter the hopping potential to all three nearest neighbors symmetrically. Efficient search algorithms can also be obtained using other types of perturbations such as a single-bond perturbation or perturbing the lattice by adding additional sites. In all cases, it is important to fine-tune the system parameters in order to operate at an avoided crossing near the Dirac point. Given the importance of graphene as a nanomaterial, our findings point towards applications in directed signal transfer, state reconstruction, or sensitive switching. This opens up the possibility of a completely new type of electronic engineering using single atoms as building blocks of electronic devices.

This work has been supported by the EPSRC network "Analysis on Graphs" (Grant No. EP/I038217/1). Helpful discussions with Klaus Richter are gratefully acknowledged.

[1] R. Portugal, Quantum Walks and Search Algorithms (Springer, New York, 2013).

[2] D. Reitzner, D. Nagaj, and V. Buzek, Acta Phys. Slovaca 61, 603 (2011).

[3] Y. Aharonov, L. Davidovich, and N. Zagury, Phys. Rev. A 48, 1687 (1993).

[4] J. Kempe, Contemp. Phys. 44, 307 (2003).

[5] V. Kendon, Phil. Trans. R. Soc. A 364, 3407 (2006).

[6] M. Santha, in Proceedings of the 5th Conference on Theory and Applications of Models of Computation (Springer, Berlin, 2008), Vol. 4978, p. 31.

[7] A. M. Childs, Phys. Rev. Lett. 102, 180501 (2009).

[8] M. Karski, L. Förster, J.-M. Choi, A. Steffen, W. Alt, D. Meschede, and A. Widera, Science 325, 174 (2009).
[9] H. Schmitz, R. Matjeschk, Ch. Schneider, J. Glueckert, M. Enderlein, T. Huber, and T. Schaetz, Phys. Rev. Lett. 103, 090504 (2009).

[10] P. Xue, B. C. Sanders, and D. Leibfried, Phys. Rev. Lett. 103, 183602 (2009).

[11] F. Zähringer, G. Kirchmair, R. Gerritsma, E. Solano, R. Blatt, and C. F. Ross, Phys. Rev. Lett. 104, 100503 (2010).

[12] H. B. Perets, Y. Lahini, F. Pozzi, M. Sorel, R. Morandotti, and Y. Silberberg, Phys. Rev. Lett. 100, 170506 (2008).

[13] H. Schreiber, K. N. Cassemiro, V. Potocek, P. J. Mosley, E. Andersson, I. Jex, and Ch. Silberhorn, Phys. Rev. Lett. 104, 050502 (2010).

[14] N. Shenvi, J. Kempe, and K. B. Whaley, Phys. Rev. A 67, 052307 (2003).

[15] L. K. Grover, in Proceedings of the 28th STOC (ACM Press, Philadelphia, 1996), pp. 212-219.

[16] L. K. Grover, Phys. Rev. Lett. 79, 325 (1997).

[17] E. Farhi and S. Gutmann, Phys. Rev. A 58, 915 (1998).

[18] A. Ambainis, J. Kempe, and A. Rivosh, in Proceedings of the Sixteenth Annual ACM-SIAM Symposium on Discrete Algorithms (SIAM, Philadelphia, 2005), pp. 1099-1108.

[19] G. Abal, R. Donangelo, F. I. Marquezino, and R. Portugal, Math. Struct. Comput. Sci. 20, 999 (2010).

[20] A. M. Childs and J. Goldstone, Phys. Rev. A 70, 022314 (2004).

[21] A. M. Childs and J. Goldstone, Phys. Rev. A 70, 042312 (2004).

[22] B. Hein and G. Tanner, Phys. Rev. Lett. 103, 260501 (2009).

[23] A. H. Castro Nero, F. Guinea, N. M. R. Peres, K. S. Novoselov, and A. K. Geim, Rev. Mod. Phys. 81, 109 (2009).

[24] H.-S. P. Wong and D. Akinwande, Carbon Nanotube and Graphene Device Physics (Cambridge University Press, Cambridge, England, 2011).

[25] F. Schedin, A. K. Geim, S. V. Morozov, E. W. Hill, P. Blake, M. I. Katsnelson, and K. S. Novoselov, Nat. Mater. 6, 652 (2007).

[26] T. O. Wehlig, K. S. Novoselov, S. V. Morozov, E. E. Vdovin, M. I. Katsnelson, A. K. Geim, and A. I. Lichtenstein, Nano Lett. 8, 173 (2008).

[27] B. Hein and G. Tanner, Phys. Rev. A 82, 012326 (2010).

[28] C. Kittel, Introduction to Solid State Physics (John Wiley \& Sons, New York, 2004), 8th ed.

[29] J. C. Meyer, C. Kisielowski, R. Erni, M. D. Rossell, M. F. Crommie, and A. Zettl, Nano Lett. 8, 3582 (2008).

[30] U. Kuhl, S. Barkhofen, T. Tudorovskiy, H.-J. Stöckmann, T. Hossain, L. de Forges de Parny, and F. Mortessagne, Phys. Rev. B 82, 094308 (2010).

[31] See Supplemental Material at http://link.aps.org/ supplemental/10.1103/PhysRevLett.112.070504 for a derivation of the logarithmic corrections to the search success amplitude and running time.

[32] L. K. Grover, Phys. Rev. Lett. 80, 4329 (1998).

[33] A. Tulsi, Phys. Rev. A 78, 012310 (2008).

[34] V. Potoček, A. Gábris, T. Kiss, and I. Jex, Phys. Rev. A 79, 012325 (2009). 